\title{
APPROXIMATION OF SOLUTION BRANCHES FOR SEMILINEAR BIFURCATION PROBLEMS
}

\author{
LAURence Cherfils ${ }^{1,2}$
}

\begin{abstract}
This note deals with the approximation, by a $P_{1}$ finite element method with numerical integration, of solution curves of a semilinear problem. Because of both mixed boundary conditions and geometrical properties of the domain, some of the solutions do not belong to $H^{2}$. So, classical results for convergence lead to poor estimates. We show how to improve such estimates with the use of weighted Sobolev spaces together with a mesh "a priori adapted" to the singularity. For the $H^{1}$ or $L^{2}$-norms, we achieve optimal results.
\end{abstract}

Résumé. Cet article concerne l'approximation, par une méthode d'éléments finis avec intégration numérique, des branches de solutions d'un problème semi-linéaire. En raison des conditions aux limites mêlées et de la géométrie du domaine, les solutions ne sont pas dans l'espace $H^{2}$. Ce qui, classiquement, entraine de mauvais taux de convergence des branches de solutions approchées vers les branches de solutions exactes. Nous montrons comment l'utilisation d'un maillage "adapté a priori" à la singularité des solutions permet d'obtenir des taux de convergence optimaux dans les normes $H^{1}$ et $L^{2}$.

AMS Subject Classification. 65N12, 65N25, 35J60.

Received: May 28, 1997. Revised: February 25, 1998.

\section{INTRODUCTION}

For $f$ given in $L^{2}(\Omega)$, let us consider the following linear and semilinear problems:

$$
\begin{aligned}
& \left\{\begin{array}{rlrl}
-\Delta u & =f & & \text { in } \Omega, \\
u & =0 & & \text { on } \Gamma_{D}, \\
\frac{\partial u}{\partial n}=0 & & \text { on } \Gamma_{N},
\end{array}\right. \\
& \left\{\begin{aligned}
-\Delta u & =\lambda u+u^{3} & & \text { in } \Omega, \\
u & =0 & & \text { on } \Gamma_{D}, \\
\frac{\partial u}{\partial n} & =0 & & \text { on } \Gamma_{N},
\end{aligned}\right.
\end{aligned}
$$

where $\Omega$, represented in Figure 1, denotes a semidisc, whose boundary $\partial \Omega$ is divided in $\Gamma_{N}=[-1,0] \times\{0\}$ and $\Gamma_{D}=\partial \Omega-\Gamma_{N}$.

Our purpose is twofold. We first want to study the convergence, towards the exact solution branches of (2), of the curves computed with a $P_{1}$ finite element method including numerical integration ( $c f$. [11]). Moreover, we also intend to illustrate, on this simple example, the use of the adaptive finite element methods for the numerical resolution of bifurcation problems with singularities (cf. [10]).

\footnotetext{
${ }^{1}$ Laboratoire LMC-IMAG, B.P. 53, 38041 Grenoble Cedex 9, France.

${ }^{2}$ Present address: Laboratoire LMCA, Université de La Rochelle, avenue Marillac, 17042 La Rochelle Cedex 1, France.
} 


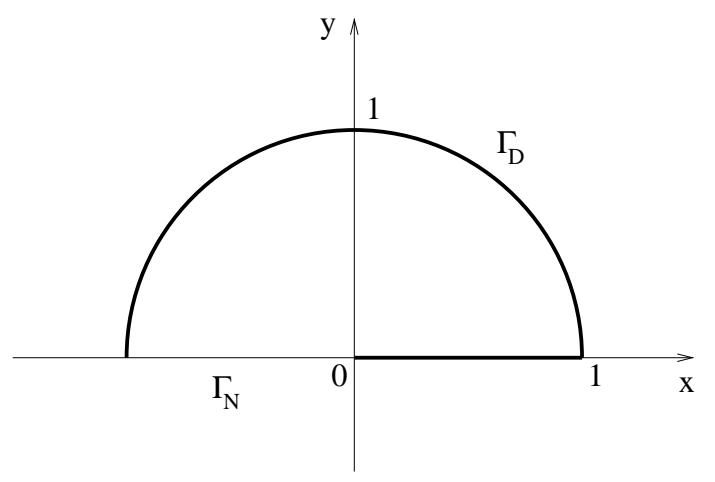

Figure 1. The domain $\Omega$.

Applying Grisvard's results [14], we know that, due to both the mixed boundary conditions and the inner angle equal to $\pi$ at the origin, the solution of problem (1) does not belong to $H^{2}(\Omega)$, but to the space $H^{\frac{3}{2}-\varepsilon}(\Omega), \varepsilon>0$. Consequently, the finite element method associated with a regular mesh will converge, in the $H^{1}$-norm, at the rate $O\left(h^{\frac{1}{2}-\varepsilon}\right.$ ) ( $c f$. Babuška-Suri [4]). Nevertheless, the a priori knowledge of the singularity permits us to overcome this lack of regularity. Classically, two strategies are possible. The first one ( $c f$. Babuška-Hoo [3], Wigley [20]...) consists in searching, on each element of the mesh, the solution in a space larger than usual, containing the singularity. The second one, consists in using a usual finite element method, posed on a strongly non-uniform mesh, "adapted to the singularity" ( $c f$. Raugel [18]). In this work, we chose the latter method, which, of course, produces a mesh closely related to those obtained with an adaptive finite element strategy.

In Section 2, we first define the non-uniform mesh which will be used further on. Then, we prove for problem (1), the convergence of the $P_{1}$ finite element method with numerical integration, in the norms of $W^{1, q}, q>2$, $H^{1}$ and $L^{2}$. For the last two, we achieve optimal rates, the same ones as for a $H^{2}$-regular solution computed on any regular mesh.

In Section 3, we show how these previously obtained convergence rates still apply to solution branches of the semilinear problem (2) (cf. Brezzi et al. [8], Crouzeix-Rappaz [13], Caloz-Rappaz [9], Paumier [16]).

Finally, we propose in Section 4, a numerical validation of one of the theoretical convergences of Section 3. These results are compared to those obtained with another kind of non uniform mesh: an a posteriori refined mesh.

First of all, setting $r(x)=\|x\|, p>1$ and $\alpha>0$, we introduce, as in Grisvard [14] and Raugel [18], the weighted Sobolev spaces defined with:

$$
W_{\alpha}^{2, p}(\Omega)=\left\{u \in W^{1, p}(\Omega) ; r^{\alpha} D^{\beta} u \in L^{p}(\Omega), \beta \in \mathbb{N}^{2},|\beta|=2\right\},
$$

and provided with the norm $\|\cdot\|_{2, p, \alpha, \Omega}$ :

$$
\|u\|_{2, p, \alpha, \Omega}^{p}=\|u\|_{1, p, \Omega}^{p}+\sum_{|\beta|=2}\left\|r^{\alpha} D^{\beta} u\right\|_{0, p, \Omega}^{p} .
$$

This space satisfies the properties, for $q>2, \frac{1}{2}<\alpha<1,1<p<\frac{2}{\alpha+1}, 2 \leq s<\frac{2 p}{2-p}$ :

(i) $W_{\alpha}^{2,2}(\Omega) \hookrightarrow W^{2, p}(\Omega) \hookrightarrow \mathcal{C}^{0}(\bar{\Omega}) \quad$ (continuous injection),

(ii) $W_{\alpha}^{2, q}(\Omega) \hookrightarrow W_{\alpha}^{2,2}(\Omega) \subset \subset W^{1, s}(\Omega) \quad$ (compact injection), 
as well as the proposition:

Proposition 1. (Raugel [18])

- Let $f \in L^{p}(\Omega), p>1$. Then $u$, solution of (1) belongs to $W_{\alpha}^{2, p}(\Omega)$, with $\alpha>\frac{3}{2}-\frac{2}{p}$,

- $\|u\|_{2, p, \alpha, \Omega} \leq c\|f\|_{0, p, \Omega}, \quad c$ constant.

\section{Convergence of the finite element method}

Let $h>0$ be a parameter which will approach 0 . Hereafter, we will mean by "optimal" mesh, a mesh composed with affine equivalent triangles with 0 belonging to the set of vertices, and satisfying the following hypotheses, for fixed $q$ and $\alpha$, with $q>2$ and $\frac{3}{2}-\frac{2}{q}<\alpha<1$.

$$
\left\{\begin{array}{l}
\left(H_{1}\right) \quad \frac{h_{K}}{\rho_{K}} \leq c_{1} \quad \forall K \in \mathcal{T}_{h}, \\
\left(H_{2}\right) \quad h_{K} \leq c_{2} h^{\frac{1}{1-\alpha}} \quad \forall K \text { having } 0 \text { as a vertex, } \\
\left(H_{3}\right) \quad h_{K} \leq c_{3} h \mathrm{~d}(0, K)^{\alpha} \quad \forall K \text { having not } 0 \text { as a vertex, } \\
\left(H_{4}\right) \quad h_{K} \geq c_{4} h^{\frac{1}{1-\alpha}} \quad \forall K \in \mathcal{T}_{h},
\end{array}\right.
$$

where $h_{K}, \rho_{K}$ and $\mathrm{d}(0, K)$ denote respectively the diameter of the triangle $K$, the diameter of the maximal ball included in $K$, and the distance between the origin and the triangle $K$. The constants $c_{i}$ are independent of $h$.

The assumption $\left(H_{1}\right)$ is classical, it only enforces the triangulation to be regular. The hypotheses $\left(H_{2}\right)$ and $\left(H_{3}\right)$ are more restrictive. They require the use of non-uniform meshes, much finer near the singularity than everywhere else. They denote the minimal assumptions necessary to obtain optimal interpolation errors in the $H^{1}$ and $L^{2}$-norms ( $c f$. Proposition 2). The last condition has been added in order to construct an inverse inequality ( $c f$. Lemma 1), weaker than the one on quasi-uniform meshes, but sufficient to prove the convergence of the finite element method in the semi-norm $|\cdot|_{1, q, \Omega}, q>2$ ( $c f$. Proposition 3).

Raugel [18] gives an example of a mesh satisfying $\left(H_{1}, H_{2}, H_{3}\right)$ (cf. fig. 3). By construction, such a mesh also satisfies the assumption $\left(H_{4}\right)$.

Thereafter, $c$ will denote a generic constant, independent of $h$. Moreover, so as to study the convergence of the $P_{1}$ finite element method with numerical integration for the resolution of problem (1), we define:

Definition 1. Let $V$ be the space $V=\left\{v \in W^{1, q}(\Omega), q>2 ; v_{/ \Gamma_{D}}=0\right\}$, and $T$ the linear operator such that for $f \in V, T f \in V \cap W_{\alpha}^{2, q}(\Omega)$ is the unique solution to:

$$
\int_{\Omega} \nabla(T f) \cdot \nabla v d x=\int_{\Omega} f v d x \quad \forall v \in H^{1}(\Omega), \quad v_{/ \Gamma_{D}}=0
$$

Let $\left(\mathcal{T}_{h}\right)_{h>0}$ be a discretization family of $\Omega$, satisfying the assumptions $\left(H_{1}\right),\left(H_{2}\right),\left(H_{3}\right),\left(H_{4}\right)$, and $\Omega_{h}$ be a polygonal convex domain such that $\underset{K \in \mathcal{T}_{h}}{\cup} K=\bar{\Omega}_{h} \subset \bar{\Omega}, \quad \partial \Omega_{h}=\Gamma_{N} \cup \Gamma_{D h}$, and the vertices of $\partial \Omega_{h}$ belong to $\partial \Omega$.

The approximation space and the test space are chosen to be:

$$
V_{h}=\left\{v_{h} \in \mathcal{C}^{0}(\bar{\Omega}) ; v_{h / K} \in P_{1}, \forall K \in \mathcal{T}_{h} ; v_{h / \Gamma_{D h}}=0 ; v_{h / \Omega-\Omega_{h}}=0\right\} .
$$

For $f \in \mathcal{C}^{0}(\bar{\Omega})$, let $T_{h} f \in V_{h}$ be the unique solution to:

$$
\int_{\Omega} \nabla\left(T_{h} f\right) . \nabla v_{h} d x=\int_{\Omega} \Pi_{h}\left(f v_{h}\right) d x \quad \forall v_{h} \in V_{h}
$$


where the $V_{h}$-interpolation operator $\Pi_{h} \in \mathcal{L}\left(\mathcal{C}^{0}(\bar{\Omega}), V_{h}\right)$ is defined for $v \in \mathcal{C}^{0}(\bar{\Omega})$ by:

$\Pi_{h} v \in V_{h}$ and $\Pi_{h} u\left(a_{i}\right)=u\left(a_{i}\right)$ for all node $a_{i}$ of $\left(\mathcal{T}_{h}\right)_{h>0}$.

We introduce two Lemmas, whose results will be useful afterwards.

Lemma 1. Let $\left(\mathcal{T}_{h}\right)_{h>0}$ be a family of triangulations satisfying the hypotheses $\left(H_{1}\right)$ and $\left(H_{4}\right)$. Let $(\alpha, q)$ be chosen to verify:

$$
2<q<\frac{8}{3} \quad ; \quad \frac{3}{2}-\frac{2}{q}<\alpha<\frac{2}{q}
$$

Thus the term $\left(1-\frac{2}{q}\right)\left(\frac{1}{1-\alpha}\right)$ is strictly less than one, and we have for $l=0$ or 1 :

$$
\left\|v_{h}\right\|_{l, q, \Omega_{h}} \leq c h^{\left(\frac{2}{q}-1\right)\left(\frac{1}{1-\alpha}\right)}\left\|v_{h}\right\|_{l, 2, \Omega_{h}} \quad \forall v_{h} \in V_{h} .
$$

Proof. Using the same arguments as Ciarlet in the proof of the inverse inequality on a quasi-uniform mesh (cf. [12]), we have, for any $K$ in $\mathcal{T}_{h}$ :

$$
\begin{aligned}
&\left|v_{h}\right|_{l, q, K} \leq c \frac{h_{K}^{l}}{\rho_{K}^{l}}(\operatorname{mes}(K))^{\frac{1}{q}-\frac{1}{2}}\left|v_{h}\right|_{l, 2, K}, \\
&\left(\operatorname{assumption}\left(H_{1}\right)\right) \leq c h_{K}^{\frac{2}{q}-1}\left|v_{h}\right|_{l, 2, K}, \\
&\left(\operatorname{assumption}\left(H_{4}\right)\right) \leq c h^{\left(\frac{2}{q}-1\right)\left(\frac{1}{1-\alpha}\right)}\left|v_{h}\right|_{l, 2, K} .
\end{aligned}
$$

So, with Jensen's inequality:

$$
\begin{aligned}
\left\{\sum_{K \in \mathcal{T}_{h}}\left|v_{h}\right|_{l, q, K}^{q}\right\}^{\frac{1}{q}} & \leq c h^{\left(\frac{2}{q}-1\right)\left(\frac{1}{1-\alpha}\right)}\left\{\sum_{K \in \mathcal{T}_{h}}\left|v_{h}\right|_{l, 2, K}^{q}\right\}^{\frac{1}{q}}, \\
& \leq c h^{\left(\frac{2}{q}-1\right)\left(\frac{1}{1-\alpha}\right)}\left\{\sum_{K \in \mathcal{T}_{h}}\left|v_{h}\right|_{l, 2, K}^{2}\right\}^{\frac{1}{2}},
\end{aligned}
$$

and we get the inverse inequality, for $l=0$ or 1 :

$$
\left|v_{h}\right|_{l, q, \Omega_{h}} \leq c h^{\left(\frac{2}{q}-1\right)\left(\frac{1}{1-\alpha}\right)}\left|v_{h}\right|_{l, 2, \Omega_{h}} \quad \forall v_{h} \in V_{h} .
$$

Moreover, for $l=1$, we deduce:

$$
\begin{aligned}
\left\|v_{h}\right\|_{1, q, \Omega_{h}}^{q} & \leq c h^{\left(\frac{2}{q}-1\right)\left(\frac{q}{1-\alpha}\right)}\left(\left|v_{h}\right|_{0,2, \Omega_{h}}^{q}+\left|v_{h}\right|_{1,2, \Omega_{h}}^{q}\right), \\
& \leq 2 c h^{\left(\frac{2}{q}-1\right)\left(\frac{q}{1-\alpha}\right)}\left\|v_{h}\right\|_{1,2, \Omega_{h}}^{q},
\end{aligned}
$$

which ends the proof of Lemma 1.

Lemma 2. With the previous notations, and $p>1$, we have:

$$
\begin{gathered}
\|u\|_{0, p, \Omega-\Omega_{h}} \leq c h\|u\|_{1, p, \Omega} \quad \forall u \in W^{1, p}(\Omega), \\
\|u\|_{1, p, \Omega-\Omega_{h}} \leq c h\|u\|_{2, p, \alpha, \Omega} \quad \forall u \in W_{\alpha}^{2, p}(\Omega) .
\end{gathered}
$$


Proof. The proof of (8) is well-known ( $c f$. Raviart-Thomas [19] for $p=2$ ). Nevertheless, this estimate can be improved, as soon as $u$ vanishes along the boundary of $\Omega$ distinct from the boundary of $\Omega_{h}$. For instance, we have, when $u \in V$ :

$$
\|u\|_{0, p, \Omega-\Omega_{h}} \leq c h^{2}\|u\|_{1, p, \Omega} \quad \forall u \in W^{1, p}(\Omega) \cap V
$$

First of all, in order to prove (9), we have to verify:

$$
\left\{\begin{array}{l}
\left\|r^{\alpha+1} \frac{\partial u}{\partial x}\right\|_{1, p, \Omega} \leq c\|u\|_{2, p, \alpha, \Omega} \\
\left\|r^{\alpha+1} \frac{\partial u}{\partial y}\right\|_{1, p, \Omega} \leq c\|u\|_{2, p, \alpha, \Omega}
\end{array}\right.
$$

The proofs of these two inequalities use the same arguments. So, we will only detail the first. We have:

$$
\left\|r^{\alpha+1} \frac{\partial u}{\partial x}\right\|_{1, p, \Omega}^{p}=\left\|r^{\alpha+1} \frac{\partial u}{\partial x}\right\|_{0, p, \Omega}^{p}+\left\|\frac{\partial}{\partial x}\left(r^{\alpha+1} \frac{\partial u}{\partial x}\right)\right\|_{0, p, \Omega}^{p}+\left\|\frac{\partial}{\partial y}\left(r^{\alpha+1} \frac{\partial u}{\partial x}\right)\right\|_{0, p, \Omega}^{p}
$$

and

$$
\left\|r^{\alpha+1} \frac{\partial u}{\partial x}\right\|_{0, p, \Omega}^{p} \leq\left\|\frac{\partial u}{\partial x}\right\|_{0, p, \Omega}^{p} \leq\|u\|_{1, p, \Omega}^{p} \leq\|u\|_{2, p, \alpha, \Omega}^{p} .
$$

Let us now study the term $\left\|\frac{\partial}{\partial x}\left(r^{\alpha+1} \frac{\partial u}{\partial x}\right)\right\|_{0, p, \Omega}^{p}$.

$$
\frac{\partial}{\partial x}\left(r^{\alpha+1} \frac{\partial u}{\partial x}\right)=\cos \theta(\alpha+1) r^{\alpha} \frac{\partial u}{\partial x}+r^{\alpha+1} \frac{\partial^{2} u}{\partial x^{2}}
$$

consequently:

$$
\begin{aligned}
\left\|\frac{\partial}{\partial x}\left(r^{\alpha+1} \frac{\partial u}{\partial x}\right)\right\|_{0, p, \Omega}^{p} & \leq c\left(\int_{\Omega}\left|\frac{\partial u}{\partial x}\right|^{p} d x d y+\int_{\Omega} r^{\alpha p}\left|\frac{\partial^{2} u}{\partial x^{2}}\right|^{p} d x d y\right) \\
& \leq c\|u\|_{2, p, \alpha, \Omega}^{p}
\end{aligned}
$$

The same results apply to the term $\left\|\frac{\partial}{\partial y}\left(r^{\alpha+1} \frac{\partial u}{\partial x}\right)\right\|_{0, p, \Omega}$, and it yields (11).

Next, applying (8) and (11) to $r^{\alpha+1} \frac{\partial u}{\partial x}$ and $r^{\alpha+1} \frac{\partial u}{\partial y}$, we obtain:

$$
\begin{aligned}
\left\|r^{\alpha+1} \frac{\partial u}{\partial x}\right\|_{0, p, \Omega-\Omega_{h}} & \leq c h\left\|r^{\alpha+1} \frac{\partial u}{\partial x}\right\|_{1, p, \Omega} \\
& \leq c h\|u\|_{2, p, \alpha, \Omega}
\end{aligned}
$$

and

Thus,

$$
\left\|r^{\alpha+1} \frac{\partial u}{\partial y}\right\|_{0, p, \Omega-\Omega_{h}} \leq c h\|u\|_{2, p, \alpha, \Omega}
$$

$$
\int_{\Omega-\Omega_{h}} r^{(\alpha+1) p}\left(\left|\frac{\partial u}{\partial x}\right|^{p}+\left|\frac{\partial u}{\partial y}\right|^{p}\right) d x d y \leq c h^{p}\|u\|_{2, p, \alpha, \Omega}^{p} .
$$

Furthermore, it can be noticed that:

$$
r>\frac{1}{2} \quad \text { sur } \Omega-\Omega_{h} .
$$


Therefore:

$$
\int_{\Omega-\Omega_{h}} r^{(\alpha+1) p}\left(\left|\frac{\partial u}{\partial x}\right|^{p}+\left|\frac{\partial u}{\partial y}\right|^{p}\right) d x d y>c|u|_{1, p, \Omega-\Omega_{h}}^{p}
$$

Then we deduce:

$$
|u|_{1, p, \Omega-\Omega_{h}} \leq c h\|u\|_{2, p, \alpha, \Omega}
$$

which, using (8), leads to (9).

Concerning the interpolation errors, we have the following results:

\section{Proposition 2.}

- Assume $u \in W^{2, p}(\Omega) \cap V, p>1$. For any family of triangulations, we have:

$$
\left\|u-\Pi_{h} u\right\|_{l, p, \Omega} \leq c h^{2-l}\|u\|_{2, p, \Omega}, \quad l=0,1 .
$$

- Assume $u \notin W^{2, q}(\Omega), u \in W_{\alpha}^{2, q}(\Omega) \cap V$ with $(\alpha, q)$ verifying (6). If the triangulation satisfies the hypotheses $\left(H_{1}\right),\left(H_{2}\right)$ and $\left(H_{3}\right)$, we have:

$$
\left\|u-\Pi_{h} u\right\|_{l, q, \Omega} \leq c h^{2-l}\|u\|_{2, q, \alpha, \Omega}, \quad l=0,1
$$

Proof. The inequality (12) for $p=2$ (resp. $p=1$ ), comes from the well-known estimate ( $c f$. Ciarlet [12]):

$$
\left\|u-\Pi_{h} u\right\|_{l, p, \Omega_{h}} \leq c h^{2-l}\|u\|_{2, p, \Omega},
$$

together with (9) (resp. (10)) and the injection $W^{2, p}(\Omega) \hookrightarrow W_{\alpha}^{2, p}(\Omega)\left(\operatorname{resp.} W^{2, p}(\Omega) \hookrightarrow W^{1, p}(\Omega)\right.$ ).

Inequalities $(9,10)$ are still convenient for dealing with $\left\|u-\Pi_{h} u\right\|_{l, q, \Omega-\Omega_{h}}$, when $u$ only belongs to $W_{\alpha}^{2, q}(\Omega) \cap V$. On the other hand, the estimate of $\left\|u-\Pi_{h} u\right\|_{l, q, \Omega_{h}}$ is more unusual. In order to justify the very restrictive assumptions on the mesh, we present here a brief description of the bound of the semi norm $\left|u-\Pi_{h} u\right|_{1, q, \Omega_{h}}$. The complete proof can be found in Raugel [18] in case $q=2$, and Cherfils [10] for $q \neq 2$.

Writing:

$$
\left|u-\Pi_{h} u\right|_{1, q, \Omega_{h}}^{q}=\sum_{K \in \mathcal{T}_{h}}\left|u-\Pi_{K} u\right|_{1, q, K}^{q},
$$

the study reduces to a local interpolation error. Because of the particular definition of the triangulation, it is clear that the term $\left|u-\Pi_{K} u\right|_{1, q, K}$ will be treated differently, whether $K$ touches the singularity or not.

case 1: 0 is a vertex of $K$

With the help of the reference element, it can be proved that $(c f .[10,18])$ :

$$
\begin{aligned}
\left|u-\Pi_{K} u\right|_{1, q, K} & \leq c \frac{h_{K}^{2}}{\rho_{K}^{1+\alpha}}|u|_{2, q, \alpha, K}, \\
\left(\operatorname{assumption}\left(H_{1}\right)\right) & \leq c h_{K}^{1-\alpha}|u|_{2, q, \alpha, K}, \\
\text { (assumption } \left.\left(H_{2}\right)\right) & \leq c h|u|_{2, q, \alpha, K} .
\end{aligned}
$$

case 2: 0 is not a vertex of $K$ 
This time, $u \in H^{2}(K)$, and we have a standard estimate (cf. [12]):

$$
\begin{aligned}
\left|u-\Pi_{K} u\right|_{1, q, K}^{q} & \leq c \frac{h_{K}^{2 q}}{\rho_{K}^{q}}|u|_{2, q, K}^{q}, \\
& \leq c h_{K}^{q}\left(\inf _{K} r^{\alpha}\right)^{-q}\left(\inf _{K} r^{\alpha}\right)^{q} \sum_{|\beta|=2} \int_{K}\left|D^{\beta} u(x)\right|^{q} d x \\
\text { (assumption } \left.\left(H_{3}\right)\right) & \leq c h^{q} \sum_{|\beta|=2} \int_{K} r^{q \alpha}\left|D^{\beta} u(x)\right|^{q} d x \\
& \leq c h^{q}|u|_{2, q, \alpha, K}^{q} .
\end{aligned}
$$

The proof of the bound of the seminorm $\left|u-\Pi_{h} u\right|_{0, q, \Omega_{h}}$ is very similar and will be omitted.

The convergence results for the finite element method including numerical integration are summarized in Proposition 3. Their proofs, relying on arguments of $[12,13,18]$, are detailed in [10].

Proposition 3. Let $\left(\mathcal{T}_{h}\right)$ be a family of triangulations satisfying the hypotheses $\left(H_{1}, \ldots, H_{4}\right)$. Let $(\alpha, q)$ be chosen to verify (6). With the notations introduced in Definition 1, we have for the finite element method with numerical integration:

$$
\begin{aligned}
\forall f \in W^{1, q}(\Omega), q>2, \quad\left\|T f-T_{h} f\right\|_{1,2, \Omega} & \leq c h\left(\|T f\|_{2,2, \alpha, \Omega}+\|f\|_{1, q, \Omega}\right), \\
\left\|T f-T_{h} f\right\|_{1, q, \Omega} & \leq c h^{\frac{2-q \alpha}{q(1-\alpha)}}\left(\|T f\|_{2, q, \alpha, \Omega}+\|f\|_{1, q, \Omega}\right), \\
\forall f \in W^{2, p}(\Omega), p>1, \quad\left\|T f-T_{h} f\right\|_{0,2, \Omega} & \leq c h^{2}\|f\|_{2, p, \Omega}, \\
\forall f \in W^{1, q}(\Omega), q>2, \quad\left\|T_{h} f\right\|_{1, q, \Omega} & \leq c\left\|\Pi_{h} f\right\|_{0,2, \Omega} .
\end{aligned}
$$

Proof. $\quad$ Let $u=T f \in V \cap W_{\alpha}^{2, q}, u_{h}=T_{h} f \in V_{h}$.

The inequality (14) is a consequence of the first Strang's Lemma (cf. Ciarlet [12]) on the one hand, and of Proposition 2, on the other hand. We have:

$$
\left\|u-u_{h}\right\|_{1,2, \Omega} \leq c\left\{\inf _{v_{h} \in V_{h}}\left\|u-v_{h}\right\|_{1,2, \Omega}+\sup _{w_{h} \in V_{h}} \frac{\left|\int_{\Omega} f w_{h} d x-\int_{\Omega} \Pi_{h}\left(f w_{h}\right) d x\right|}{\left\|w_{h}\right\|_{1,2, \Omega}}\right\}
$$

As usual, the term relevant to numerical integration converges at the rate $O(h)(c f .[12])$. Using Propositions 2 and 1 , we conclude:

$$
\begin{aligned}
\left\|u-u_{h}\right\|_{1,2, \Omega} & \leq c\left\{\left\|u-\Pi_{h} u\right\|_{1,2, \Omega}+h\|f\|_{1, q, \Omega}\right\} \\
& \leq c h\left(\|u\|_{2,2, \alpha, \Omega}+\|f\|_{1, q, \Omega}\right) \\
& \leq c h\|f\|_{1, q, \Omega} .
\end{aligned}
$$


Next, in order to verify (15), we apply Lemma 1, Proposition 2 and inequality (14). Thus we get:

$$
\begin{aligned}
\left\|u-u_{h}\right\|_{1, q, \Omega} & \leq\left\|u-\Pi_{h} u\right\|_{1, q, \Omega}+\left\|\Pi_{h} u-u_{h}\right\|_{1, q, \Omega_{h}}, \\
& \leq c h\|u\|_{2, q, \alpha, \Omega}+c h^{\left(\frac{2}{q}-1\right)\left(\frac{1}{1-\alpha}\right)}\left\|\Pi_{h} u-u_{h}\right\|_{1,2, \Omega_{h}}, \\
& \leq c h\|u\|_{2, q, \alpha, \Omega}+c h^{\left(\frac{2}{q}-1\right)\left(\frac{1}{1-\alpha}\right)}\left(\left\|\Pi_{h} u-u\right\|_{1,2, \Omega_{h}}+\left\|u-u_{h}\right\|_{1,2, \Omega_{h}}\right), \\
& \leq c h^{\frac{2-q \alpha}{q(1-\alpha)}}\left(\|u\|_{2, q, \alpha, \Omega}+\|f\|_{1, q, \Omega}\right) .
\end{aligned}
$$

Our proof for (16) follows from the one of Crouzeix-Rappaz [13]. First, we notice that the operators $T_{h}$ and $\Pi_{h}$ satisfy:

$$
T_{h} f=T_{h}\left(\Pi_{h} f\right) \quad \forall f \in \mathcal{C}^{0}(\bar{\Omega}) .
$$

We set $u_{h}=T_{h} f=T_{h}\left(\Pi_{h} f\right), w=T\left(\Pi_{h} f\right)$ and $w_{h}=T_{h}^{\prime}\left(\Pi_{h} f\right)$, where $T_{h}^{\prime}$ is a linear operator defined by:

for all $g \in L^{2}(\Omega), T_{h}^{\prime} g \in V_{h}$ is the unique solution to

$$
\int_{\Omega} \nabla\left(T_{h}^{\prime} g\right) \cdot \nabla v_{h} d x=\int_{\Omega} g v_{h} d x \quad \forall v_{h} \in V_{h}
$$

The error $\left\|u-u_{h}\right\|_{0,2, \Omega}$ may be decomposed into 3 terms which will be treated separately:

$$
\left\|u-u_{h}\right\|_{0,2, \Omega} \leq\|u-w\|_{0,2, \Omega}+\left\|w-w_{h}\right\|_{0,2, \Omega}+\left\|w_{h}-u_{h}\right\|_{0,2, \Omega} .
$$

The term $\left\|w-w_{h}\right\|_{0,2, \Omega}$ is in fact relevant to the convergence of the finite element method without numerical integration. As usual, we use Aubin-Nitsche's Lemma ( $c f .[12])$, which can be written in our particular case:

$$
\left\|w-w_{h}\right\|_{0,2, \Omega} \leq c\left\|w-w_{h}\right\|_{1,2, \Omega}\left(\sup _{g \in L^{2}(\Omega)}\left\{\frac{1}{\|g\|_{0,2, \Omega}} \inf _{v_{h} \in V_{h}}\left\|T g-v_{h}\right\|_{1,2, \Omega}\right\}\right)
$$

Moreover, using Propositions 1 and 2,

$$
\inf _{v_{h} \in V_{h}}\left\|T g-v_{h}\right\|_{1,2, \Omega} \leq\left\|T g-\Pi_{h} T g\right\|_{1,2, \Omega} \leq c h\|T g\|_{2,2, \alpha, \Omega} \leq c h\|g\|_{0,2, \Omega}
$$

Thus, combining $(19,20)$ and Céa's Lemma:

$$
\begin{aligned}
\left\|w-w_{h}\right\|_{0,2, \Omega} & \leq c h\left\|w-w_{h}\right\|_{1,2, \Omega} \\
& \leq c h^{2}\|w\|_{2,2, \alpha \Omega} .
\end{aligned}
$$

Taking into account that $W^{2, p}(\Omega) \hookrightarrow \mathcal{C}^{0}(\bar{\Omega})$, when $p>1$, it follows that:

$$
\begin{aligned}
\left\|w-w_{h}\right\|_{0,2, \Omega} & \leq c h^{2}\left\|T \Pi_{h} f\right\|_{2,2, \alpha, \Omega}, \\
& \leq c h^{2}\left\|\Pi_{h} f\right\|_{0,2, \Omega}, \\
& \leq c h^{2}\|f\|_{2, p, \Omega} .
\end{aligned}
$$


Next, to deal with the term $\|u-w\|_{0,2, \Omega}$, we use Propositions 1 and 2, and the Sobolev imbedding $W_{\alpha}^{2, p}(\Omega) \hookrightarrow$ $W^{1, p}(\Omega) \hookrightarrow L^{2}(\Omega)$. It yields:

$$
\begin{aligned}
\|u-w\|_{0,2, \Omega} & =\left\|T f-T \Pi_{h} f\right\|_{0,2, \Omega}, \\
& \leq\left\|T f-T \Pi_{h} f\right\|_{2, p, \alpha, \Omega}, \\
\text { (Proposition 1) } & \leq\left\|f-\Pi_{h} f\right\|_{0, p, \Omega} \\
\text { (Proposition 2) } & \leq c h^{2}\|f\|_{2, p, \Omega} .
\end{aligned}
$$

Finally, to handle the last term $\left\|w_{h}-u_{h}\right\|_{0,2, \Omega}$, we need the following result, whose proof is very similar to that of Crouzeix-Rappaz [13] and will be omitted,

$$
\left|w_{h}-u_{h}\right|_{1,2, \Omega} \leq c h^{2}\left|\Pi_{h} f\right|_{1,2, \Omega} .
$$

Thus we get, since $H^{1}$-norm and $H^{1}$-seminorm are equivalent on $V$ :

$$
\begin{aligned}
\left\|w_{h}-u_{h}\right\|_{0,2, \Omega} & \leq\left\|w_{h}-u_{h}\right\|_{1,2, \Omega} \\
& \leq c\left|w_{h}-u_{h}\right|_{1,2, \Omega} \\
& \leq c h^{2}\left|\Pi_{h} f\right|_{1,2, \Omega}, \\
& \leq c h^{2}\|f\|_{2, p, \Omega} .
\end{aligned}
$$

Combining the above results, we get (16).

For the proof of (17), we write:

$$
\left\|u_{h}\right\|_{1, q, \Omega} \leq\left\|u_{h}-w_{h}\right\|_{1, q, \Omega}+\left\|w_{h}-w\right\|_{1, q, \Omega}+\|w\|_{1, q, \Omega}
$$

Classically, we have (see [13]):

$$
\left|u_{h}-w_{h}\right|_{1,2, \Omega} \leq c h\left\|\Pi_{h} f\right\|_{0,2, \Omega} .
$$

Thus, applying Lemma 1, we get:

$$
\begin{aligned}
\left\|u_{h}-w_{h}\right\|_{1, q, \Omega}=\left\|u_{h}-w_{h}\right\|_{1, q, \Omega_{h}} & \leq c h^{\left(\frac{2}{q}-1\right)\left(\frac{1}{1-\alpha}\right)}\left\|u_{h}-w_{h}\right\|_{1,2, \Omega_{h}}, \\
& \leq c h^{\left(\frac{2}{q}-1\right)\left(\frac{1}{1-\alpha}\right)}\left|u_{h}-w_{h}\right|_{1,2, \Omega_{h}}, \\
& \leq c h^{\frac{2-q \alpha}{q(1-\alpha)}}\left\|\Pi_{h} f\right\|_{0,2, \Omega}, \\
& \leq c\left\|\Pi_{h} f\right\|_{0,2, \Omega} .
\end{aligned}
$$

Since the term $\left\|w-w_{h}\right\|_{0,2, \Omega}$ is relevant to the convergence of the finite element method without numerical integration, the inequality (14) reduces to:

$$
\left\|w-w_{h}\right\|_{0,2, \Omega} \leq c h\|w\|_{2,2, \alpha, \Omega_{h}} .
$$




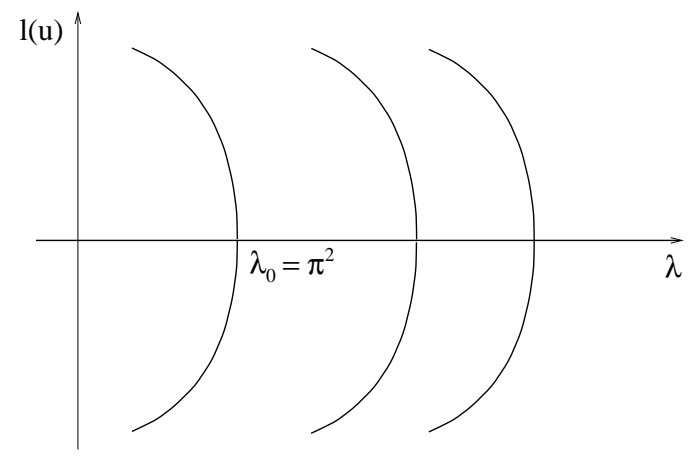

Figure 2. Bifurcation diagram for the problem (22).

Thus, using Lemma 1 and Proposition 2, we have:

$$
\begin{aligned}
\left\|w_{h}-w\right\|_{1, q, \Omega} & \leq\left\|w_{h}-\Pi_{h} w\right\|_{1, q, \Omega}+\left\|\Pi_{h} w-w\right\|_{1, q, \Omega} \\
& \leq c h^{\left(\frac{2}{q}-1\right)\left(\frac{1}{1-\alpha}\right)}\left\|w_{h}-\Pi_{h} w\right\|_{1,2, \Omega_{h}}+c h\|w\|_{2, q, \alpha, \Omega}, \\
& \leq c h^{\frac{2-q \alpha}{q(1-\alpha)}}\|w\|_{2,2, \alpha, \Omega}+c h\left\|\Pi_{h} f\right\|_{0, q, \Omega}, \\
& \leq c h^{\frac{2-q \alpha}{q(1-\alpha)}}\left\|\Pi_{h} f\right\|_{0,2, \Omega}+c h^{\frac{2-q \alpha}{q(1-\alpha)}}\left\|\Pi_{h} f\right\|_{0,2, \Omega}, \\
& \leq c\left\|\Pi_{h} f\right\|_{0,2, \Omega} .
\end{aligned}
$$

Finally, we have:

$$
\begin{aligned}
\|w\|_{1, q, \Omega} & \leq\|w\|_{2,2, \alpha, \Omega}, \\
& \leq c\left\|\Pi_{h} f\right\|_{0,2, \Omega} .
\end{aligned}
$$

The above bounds applied to (21) lead to (17).

Remark 1. Our estimate (15) is not optimal. Setting $\alpha=0$ in (15), it leads to a convergence with the rate $O\left(h^{\frac{2}{q}}\right)$ for a regular solution (i.e. belonging to $H^{2}(\Omega)$ ), instead of $O(h)$, which, according to Rannacher and Scott's results [17], is the optimal rate. Nevertheless, as it will be pointed out later, our single aim is, in order to apply Theorem 1 , to prove that the finite element method converges in the norm $\|\cdot\|_{1, q, \Omega}$, for $(\alpha, q)$ satisfying (6).

\section{Approximation of a SEMilineAR BIFURCATION PROBLEM}

Now, we consider the semilinear problem:

$$
\left\{\begin{aligned}
-\Delta u & =\lambda u+u^{3} & & \text { in } \Omega, \\
u & =0 & & \text { on } \Gamma_{D}, \\
\frac{\partial u}{\partial n} & =0 & & \text { on } \Gamma_{N} .
\end{aligned}\right.
$$

According to Brezzi et al. [8], the solution branches of (22) look as in Figure 2. Our purpose in this Section is to estimate the error induced by the computation of a nontrivial solution branch near a bifurcation point. We use for the computation a $P_{1}$ finite element method with numerical integration. 
First of all, the problem (22) is written in a standard way for bifurcation problems (cf. [8]):

$$
\left\{\begin{array}{c}
\text { find }(\lambda, u) \in \mathbb{R} \times V \\
F_{0}(\lambda, u)=u+T G(\lambda, u)=0
\end{array}\right.
$$

with:

- $V, T$ defined as in Definition 1 , and $q>2$ satisfying (6),

- $G:\left\{\begin{aligned} \mathbb{R} \times V & \longrightarrow V \\ (\lambda, u) & \longrightarrow-\lambda u-u^{3} .\end{aligned}\right.$

The operator $T$ is linear, continuous from $L^{p}(\Omega)$ into $W_{\alpha}^{2, p}(\Omega)$, for $p>1$ and $\alpha>\frac{3}{2}-\frac{2}{p}$ (Proposition 1). It is also straightforward to verify that $T$ is compact from $V$ into $V$, and self-adjoint on $H^{1}(\Omega)$. Moreover, the mapping $G$, and consequently $F$, are $\mathcal{C}^{\infty}$ from $\mathbb{R} \times V$ into $V$.

Hereafter, we will assume that $\left(\lambda_{0}, 0\right)$ is a simple bifurcation point of $(23)$ (cf. [8]). This means that:

$$
V=N\left(D_{u} F^{0}\right) \oplus R\left(D_{u} F^{0}\right),
$$

and the existence of:

- $\varphi_{0} \in V$, such that $N\left(D_{u} F^{0}\right)=\mathbb{R} \varphi_{0}$ and $\left\|\varphi_{0}\right\|_{V}=1$,

- $\varphi_{0}^{*} \in V^{\prime}$, such that $N\left(D_{u} F^{0 *}\right)=\mathbb{R} \varphi_{0}^{*}\left(=R\left(D_{u} F^{0}\right)^{\perp}\right)$ and $\left\langle\varphi_{0}^{*}, \varphi_{0}\right\rangle_{V^{\prime}, V}=1$,

where $D_{u} F^{0}=D_{u} F_{0}\left(\lambda_{0}, 0\right)$, and $N\left(D_{u} F^{0}\right), R\left(D_{u} F^{0}\right)$ are respectively the kernel and the range of the derivative $D_{u} F^{0}$. The function $\varphi_{0}$ is in fact an eigenfunction of the operator $T$, associated with the eigenvalue $\frac{-1}{\lambda_{0}}$.

Besides, let $\left(\lambda_{0}, 0\right)$ be such that $\varphi_{0} \notin H^{2}(\Omega)$ (this is true for example if $\left(\lambda_{0}, 0\right)$ is the first bifurcation point of $(22), c f .[10])$.

The trivial branch being solution of $(23)$, the non trivial one may be, near the bifurcation point $\left(\lambda_{0}, 0\right)$, parametrized with, for $|\alpha| \leq \alpha_{0}$, (cf. Brezzi al. [8], Crouzeix-Rappaz [13], Paumier [16]):

$$
\left\{\begin{array}{l}
\underline{\lambda}_{0}(\alpha)=\lambda_{0}+\xi(\alpha), \\
\underline{u}_{0}(\alpha)=\alpha \varphi_{0}+v(\alpha, \xi(\alpha)),
\end{array}\right.
$$

with $v:\left[-\alpha_{0}, \alpha_{0}\right] \times \mathbb{R} \mapsto R\left(D_{u} F^{0}\right), \xi:\left[-\alpha_{0}, \alpha_{0}\right] \mapsto \mathbb{R}$ such that $\xi(0)=0, v(0,0)=0$, and the application $\alpha \longmapsto\left(\underline{\lambda}_{0}(\alpha), \underline{u}_{0}(\alpha)\right)$ of class $\mathcal{C}^{\infty}$ from $\left[-\alpha_{0}, \alpha_{0}\right]$ into $V$.

Consequently, we have:

$$
\left\langle\varphi_{0}^{*}, \underline{u}_{0}(\alpha)\right\rangle_{V^{\prime}, V}=\alpha \quad \forall \alpha \leq\left|\alpha_{0}\right| .
$$

The error induced by the numerical approximation of the non trivial branch

$$
\mathcal{B}=\left\{\left(\underline{\lambda}_{0}(\alpha), \underline{u}_{0}(\alpha)\right) ;|\alpha| \leq \alpha_{0}\right\}
$$

comes from Paumier's results [16]:

Theorem 1. Under the assumptions:

$$
\left\{\begin{array}{l}
V_{h} \text { is a finite dimensional subspace of } V \\
T_{h} \in \mathcal{L}\left(V ; V_{h}\right), \\
\lim _{h \rightarrow 0}\left\|T-T_{h}\right\|_{\mathcal{L}(V ; V)}=0
\end{array}\right.
$$


there exists $h_{0}>0$ and $r>0$ such that:

$\forall h \in\left[0, h_{0}\right]$, the set of the solutions of

$$
\left\{\begin{array}{c}
F_{h}: \mathbb{R} \times V_{h} \rightarrow V_{h} \\
F_{h}(\lambda, u)=0=u+T_{h} G(\lambda, u)
\end{array}\right.
$$

included in the ball $B_{r}\left(\lambda_{0}, 0\right) \subset \mathbb{R} \times V$, contains two branches: the branch $\mathcal{B}_{h}=$ $\left\{\left(\underline{\lambda}_{h}(\alpha), \underline{u}_{h}(\alpha)\right) ;|\alpha| \leq \alpha_{0}\right\}$ also solutions of (24), and the trivial branch, which intersect at a simple bifurcation point $\left(\lambda_{h}^{0}, 0\right)$, with $\underline{\lambda}_{h}(0)=\lambda_{h}^{0}$.

Furthermore, $\forall j \in \mathbb{N}$, there exists $c>0$ such that $\forall h \in\left[0, h_{0}\right], \forall(\underline{\lambda}, \underline{u}) \in B_{r}\left(\underline{\lambda}_{0}, \underline{u}_{0}\right) \subset \mathcal{C}^{\infty}\left(\left[-\alpha_{0}, \alpha_{0}\right], \mathbb{R} \times\right.$ $V)$, satisfying $\underline{u}(0)=0$, we have (for $\left.|\alpha| \leq \alpha_{0}\right)$ :

$$
\begin{aligned}
& \left|D_{\alpha}^{j} \underline{\lambda}(\alpha)-D_{\alpha}^{j} \underline{\lambda}_{h}(\alpha)\right|+\left\|D_{\alpha}^{j} \underline{u}(\alpha)-D_{\alpha}^{j} \underline{u}_{h}(\alpha)\right\|_{V} \\
& \leq c \sum_{i=1}^{j+1}\left\{\sup _{|t| \leq|\alpha|}\left|D_{t}^{i}\left\{\left\langle\varphi_{0}^{*}, \underline{u}(t)\right\rangle_{V^{\prime}, V}-t\right\}\right|+\sup _{|t| \leq|\alpha|}\left\|D_{t}^{i} F_{h}(\underline{\lambda}(t), \underline{u}(t))\right\|_{V}\right\} .
\end{aligned}
$$

(we use the notation $D_{t}^{i}=\frac{d^{i}}{d t^{i}}$, and $D_{\alpha}^{i}=\frac{d^{i}}{d \alpha^{i}}$.)

We apply Theorem 1 to our example and choose $\mathcal{T}_{h}, V_{h}$, and $T_{h}$ as introduced in Definition 1 . So, Proposition 3 yields to:

$$
\left\|T-T_{h}\right\|_{\mathcal{L}(V ; V)} \leq c h^{\frac{2-q \alpha}{q(1-\alpha)}} \stackrel{h}{\rightarrow}{ }^{0} 0,
$$

and the assumptions (25) are verified.

Applying (27) with $\underline{\lambda}=\underline{\lambda}_{0}, \underline{u}=\underline{u}_{0}$, we achieve the rate $O\left(h^{\frac{2-q \alpha}{q(1-\alpha)}}\right)$ for the error in the norm of $V$. However, this estimate may be improved by a more suitable choice of $(\underline{\lambda}, \underline{u})(c f .[9,13])$, i.e. $\underline{\lambda}=\underline{\lambda}_{0}$ and $\underline{u}=\underline{\tilde{u}}_{h}=$ $-T_{h} G\left(\underline{\lambda}_{0}, \underline{u}_{0}\right)$. It can be easily verified that $\left(\underline{\lambda}_{0}, \underline{\tilde{u}}_{h}\right)$ belongs to the ball $B_{r}\left(\underline{\lambda}_{0}, \underline{u}_{0}\right)$. Applying (27), we get:

Lemma 3. With the previous notations, we have, for $|\alpha| \leq \alpha_{0}$ :

$$
\left|D_{\alpha}^{j} \underline{\lambda}_{0}(\alpha)-D_{\alpha}^{j} \underline{\lambda}_{h}(\alpha)\right|+\left\|D_{\alpha}^{j} \underline{\tilde{u}}_{h}(\alpha)-D_{\alpha}^{j} \underline{u}_{h}(\alpha)\right\|_{V} \leq c h^{2}
$$

$c$ being a constant independent of $h$ and $\alpha$.

Proof. We begin to study the first part of the right hand side of (27). We have:

$$
\begin{aligned}
D_{t}^{i}\left(\left\langle\varphi_{0}^{*}, \underline{\tilde{u}}_{h}(t)\right\rangle_{V^{\prime}, V}-t\right) & =D_{t}^{i}\left(\left\langle\varphi_{0}^{*}, \underline{\tilde{u}}_{h}(t)-\underline{u}_{0}(t)\right\rangle_{V^{\prime}, V}\right), \\
& =\left\langle\varphi_{0}^{*},\left(T-T_{h}\right) D_{t}^{i} G\left(\underline{\lambda}_{0}(t), \underline{u}_{0}(t)\right)\right\rangle_{V^{\prime}, V}
\end{aligned}
$$

The operator $T$ is self-adjoint from $H^{1}(\Omega)$ into itself. So, with the imbedding $W^{1, q}(\Omega) \hookrightarrow H^{1}(\Omega) \hookrightarrow\left(W^{1, q}\right)^{\prime}(\Omega)$, we deduce that $\varphi_{0}^{*}$ has the form $(c f .[15])$ :

$$
V \ni v \longmapsto\left|\varphi_{0}\right|_{1,2, \Omega}^{-2} \int_{\Omega} \nabla \varphi_{0} \nabla v d x d y
$$


Consequently, it yields:

$$
\begin{aligned}
\left|D_{t}^{i}\left(\left\langle\varphi_{0}^{*}, \underline{\tilde{u}}_{h}(t)\right\rangle_{V^{\prime}, V}-t\right)\right| & =\frac{1}{\left|\varphi_{0}\right|_{1,2, \Omega}^{2}}\left|\int_{\Omega} \nabla \varphi_{0} \nabla\left(T-T_{h}\right) D_{t}^{i} G\left(\underline{\lambda}_{0}(t), \underline{u}_{0}(t)\right) d x d y\right|, \\
& =\frac{\left|\lambda_{0}\right|}{\left|\varphi_{0}\right|_{1,2, \Omega}^{2}}\left|\int_{\Omega} \varphi_{0}\left(T-T_{h}\right) D_{t}^{i} G\left(\underline{\lambda}_{0}(t), \underline{u}_{0}(t)\right) d x d y\right|, \\
& \leq c\left\|\left(T-T_{h}\right) D_{t}^{i} G\left(\underline{\lambda}_{0}(t), \underline{u}_{0}(t)\right)\right\|_{0,2, \Omega} .
\end{aligned}
$$

Moreover, $\underline{u}_{0}(t) \in W_{\alpha}^{2,2}(\Omega)$. Thus there exists $p_{0}>1$, such that the imbedding $W_{\alpha}^{2,2}(\Omega) \hookrightarrow W^{2, p_{0}}(\Omega)$ hold. The space $W^{2, p_{0}}(\Omega)$ being a Banach algebra $(c f .[1])$, it follows that $G\left(\underline{\lambda}_{0}(t), \underline{u}_{0}(t)\right) \in W^{2, p_{0}}(\Omega)$.

The Proposition 3 leads to:

$$
\left|D_{t}^{i}\left(\left\langle\varphi_{0}^{*}, \underline{\tilde{u}}_{h}(t)\right\rangle_{V^{\prime}, V}-t\right)\right| \leq c h^{2}\left\|D_{t}^{i} G\left(\underline{\lambda}_{0}(t), \underline{u}_{0}(t)\right)\right\|_{2, p_{0}, \Omega} .
$$

Next, we have to study the term $\left\|D_{t}^{i} F_{h}\left(\underline{\lambda}_{0}(t), \underline{\tilde{u}}_{h}(t)\right)\right\|_{V}$, occuring in (27). Our arguments are classical $(c f$. Crouzeix-Rappaz [13], Caloz-Rappaz [9], ... ).

Thanks to (17), we have:

$$
\begin{aligned}
\left\|D_{t}^{i} F_{h}\left(\underline{\lambda}_{0}(t), \underline{\tilde{u}}_{h}(t)\right)\right\|_{V} & \leq\left\|T_{h} D_{t}^{i}\left(G\left(\underline{\lambda}_{0}(t), \underline{\tilde{u}}_{h}(t)\right)-G\left(\underline{\lambda}_{0}(t), \underline{u}_{0}(t)\right)\right)\right\|_{1, q, \Omega}, \\
& \leq c\left\|\Pi_{h} D_{t}^{i}\left\{A_{h}(t)\left(\underline{\tilde{u}}_{h}(t)-\underline{u}_{0}(t)\right)\right\}\right\|_{0,2, \Omega},
\end{aligned}
$$

with:

$$
A_{h}(t)=\int_{0}^{1} D_{u} G\left(\underline{u}_{0}(t)+s\left(\underline{\tilde{u}}_{h}(t)-\underline{u}_{0}(t)\right)\right) d s .
$$

Furthermore, we can verify that the terms $D_{t}^{i} A_{h}(t)$ are uniformly bounded. Since for all $a, g \in \mathcal{C}^{0}(\bar{\Omega})$, $\left\|\Pi_{h}(a g)\right\|_{0,2, \Omega} \leq c\|a\|_{0, \infty, \Omega}\left\|\Pi_{h} g\right\|_{0,2, \Omega}$, we have:

$$
\begin{aligned}
\sum_{i=1}^{j+1}\left\|D_{t}^{i} F_{h}\left(\underline{\lambda}_{0}(t), \underline{\tilde{u}}_{h}(t)\right)\right\|_{V} & \leq c \sum_{i=0}^{j+1}\left\|\Pi_{h} D_{t}^{i}\left(\underline{\tilde{u}}_{h}(t)-\underline{u}_{0}(t)\right)\right\|_{0,2, \Omega}, \\
& \leq c \sum_{i=0}^{j+1}\left\|\left(\Pi_{h} T-T_{h}\right) D_{t}^{i} G\left(\underline{\lambda}_{0}(t), \underline{u}_{0}(t)\right)\right\|_{0,2, \Omega} .
\end{aligned}
$$

Again, we conclude with Propositions 2 and 3:

$$
\sum_{i=1}^{j+1}\left\|D_{t}^{i} F_{h}\left(\underline{\lambda}_{0}(t), \underline{\tilde{u}}_{h}(t)\right)\right\|_{V} \leq c h^{2} \sum_{i=0}^{j+1}\left\|D_{t}^{i} G\left(\underline{\lambda}_{0}(t), \underline{u}_{0}(t)\right)\right\|_{2, p_{0}, \Omega} .
$$

Finally, we have to make sure that the terms $\left\|D_{t}^{i} G\left(\underline{\lambda}_{0}(t), \underline{u}_{0}(t)\right)\right\|_{2, p_{0}, \Omega}$ are bounded for all $t \in[-\alpha, \alpha]$. Since the map $t \longmapsto\left(\underline{\lambda}_{0}(t), \underline{u}_{0}(t)\right)$ is continuous from the compact $\left[-\alpha_{0}, \alpha_{0}\right]$ into $V$, we have:

$$
\sup _{|t| \leq|\alpha|}\left\{\left|\underline{\lambda}_{0}(t)\right|+\left\|\underline{u}_{0}(t)\right\|_{V}\right\} \leq M,
$$

where $M$ denotes a constant independent of $\alpha$. 
Furthermore, $W^{2, p_{0}}(\Omega)$ being a Banach algebra, it is sufficient to note:

$$
\begin{aligned}
\left\|\underline{u}_{0}(t)\right\|_{2, p_{0}, \Omega} & =\left\|T G\left(\underline{\lambda}_{0}(t), \underline{u}_{0}(t)\right)\right\|_{2, p_{0}, \Omega}, \\
& \leq c\left\|T G\left(\underline{\lambda}_{0}(t), \underline{u}_{0}(t)\right)\right\|_{2,2, \alpha, \Omega}, \\
\text { (Proposition 1) } & \leq c\left\|G\left(\underline{\lambda}_{0}(t), \underline{u}_{0}(t)\right)\right\|_{1, q, \Omega}, \\
& \leq c\left(M^{2}+M^{3}\right),
\end{aligned}
$$

to conclude that $\left\|G\left(\underline{\lambda}_{0}(t), \underline{u}_{0}(t)\right)\right\|_{2, p_{0}, \Omega}$ is bounded on $[-\alpha, \alpha]$. Such arguments apply to all terms $\left\|D_{t}^{i} G\left(\underline{\lambda}_{0}(t), \underline{u}_{0}(t)\right)\right\|_{2, p_{0}, \Omega}$, for $i \in \mathbb{N}$. This, combined with (27), (28), (29), ends the proof of Lemma 3 .

We can now state the main result of this note:

Theorem 2. Let $\mathcal{T}_{h}$ be a triangulation satisfying assumptions $\left(H_{1}\right),\left(H_{2}\right),\left(H_{3}\right),\left(H_{4}\right)$. Let also the exact and approached bifurcation problem be defined as previously. For $j \in \mathbb{N},|\alpha| \leq \alpha_{0}$, and c a generic constant independent of $h$ and $\alpha$, we have:

$$
\begin{aligned}
\left\|D_{\alpha}^{j} \underline{u}_{0}(\alpha)-D_{\alpha}^{j} \underline{u}_{h}(\alpha)\right\|_{1,2, \Omega} & \leq c h, \\
\left\|D_{\alpha}^{j} \underline{u}_{0}(\alpha)-D_{\alpha}^{j} \underline{u}_{h}(\alpha)\right\|_{0,2, \Omega} & \leq c h^{2}, \\
\left|D_{\alpha}^{j} \underline{\lambda}_{0}(\alpha)-D_{\alpha}^{j} \underline{\lambda}_{h}(\alpha)\right| & \leq c h^{2} .
\end{aligned}
$$

Proof. The estimates in Theorem 2 follow from Lemma 3. We just need to notice that, for $l=0$ or 1 :

$$
\begin{aligned}
& \left|D_{\alpha}^{j} \underline{\lambda}_{0}(\alpha)-D_{\alpha}^{j} \underline{\lambda}_{h}(\alpha)\right|+\left\|D_{\alpha}^{j} \underline{u}_{0}(\alpha)-D_{\alpha}^{j} \underline{u}_{h}(\alpha)\right\|_{l, 2, \Omega} \\
& \leq\left|D_{\alpha}^{j} \underline{\lambda}_{0}(\alpha)-D_{\alpha}^{j} \underline{\lambda}_{h}(\alpha)\right|+\left\|D_{\alpha}^{j} \underline{u}_{0}(\alpha)-D_{\alpha}^{j} \underline{\tilde{u}}_{h}(\alpha)\right\|_{l, 2, \Omega}+\left\|D_{\alpha}^{j} \underline{\tilde{u}}_{h}(\alpha)-D_{\alpha}^{j} \underline{u}_{h}(\alpha)\right\|_{1, q, \Omega}, \\
& \leq c h^{2}+\left\|D_{\alpha}^{j} \underline{u}_{0}(\alpha)-D_{\alpha}^{j} \underline{\tilde{u}}_{h}(\alpha)\right\|_{l, 2, \Omega}, \\
& \leq c h^{2}+\left\|\left(T-T_{h}\right) D_{\alpha}^{j} G\left(\underline{\lambda}_{0}(\alpha), \underline{u}_{0}(\alpha)\right)\right\|_{l, 2, \Omega} .
\end{aligned}
$$

Once again, we conclude with Proposition 3.

\section{Numerical RESUlts}

The main purpose of this Section is to validate the theoretical estimate (32) in Theorem 2, in the case of the first bifurcation point, with $\alpha=0=j$. Nevertheless, we also intend to point out the efficiency of the adaptive finite element method for general bifurcation problems with singularities. So, we compare our convergence results obtained on an "optimal" mesh (Fig. 3), with those obtained on two other kinds of meshes: a uniform and an adapted one. In the latter case, the adaptive procedure is made of a succession of mesh refinements, resulting from local a posteriori error estimates (Fig. 4). The solutions of (22), eigenvalues and eigenfunctions, are computed with a continuation method (cf. [2], and [10] in the adaptive case).

It can be seen from Figure 5 that the convergence rate $O\left(h^{2}\right)$ for the "optimal mesh" has effectively been obtained. Nonetheless, on this example, the results with the a posteriori refined meshes are also satisfactory. Adaptive finite element methods have the property of producing a mesh close to the optimal thus leading to a good convergence, without requiring, for the implementation, any a priori information about the singularity. So, 


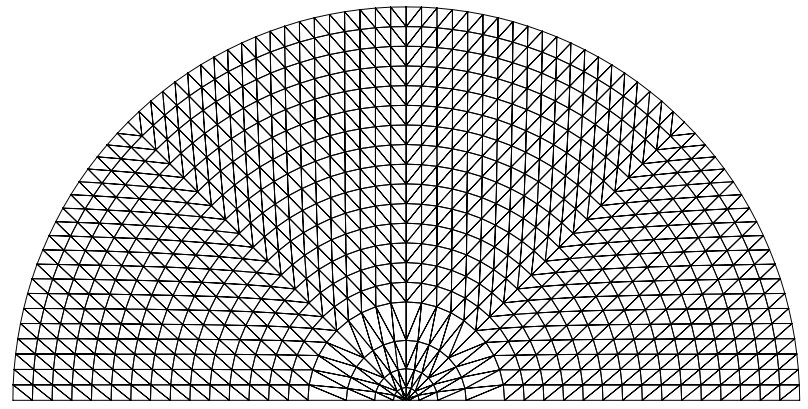

Figure 3. Example of a mesh satisfying assumptions $\left(H_{1}\right) \ldots\left(H_{4}\right)$, with $\alpha=0.55$.
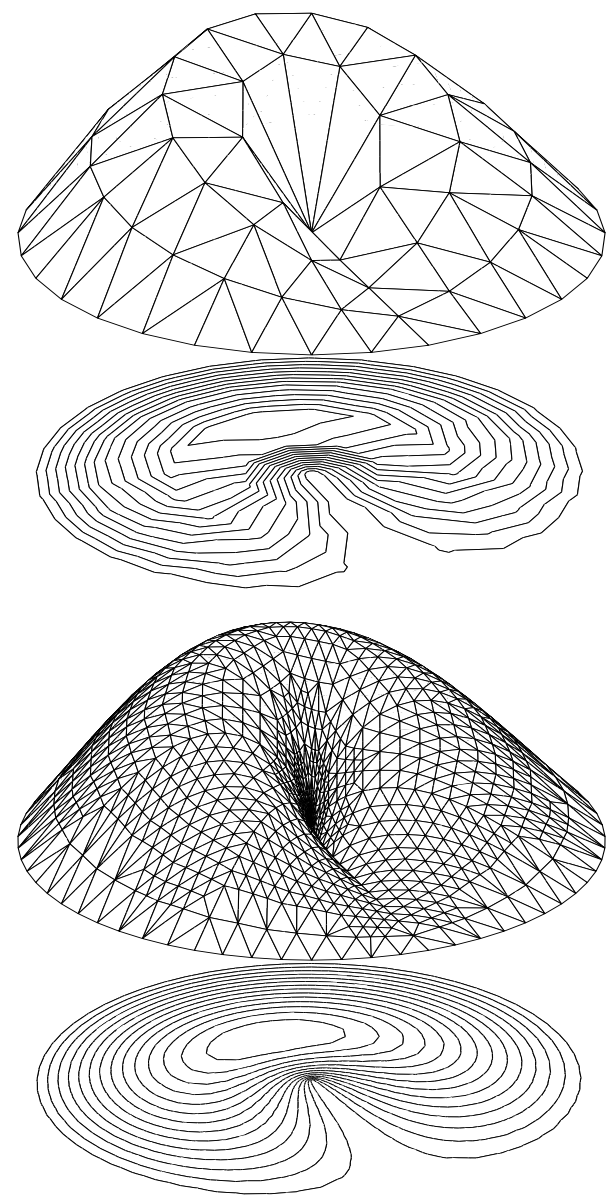

FiguRE 4. Coarse and refined solution of (26), represented by symmetry on the entire disc.

they seem to be well suited to bifurcation problems with boundary layers or unknown singularities. Moreover, we notice from Figure 6 that, at least for this example, refined meshes do not involve more degrees of freedom than the optimal ones. 


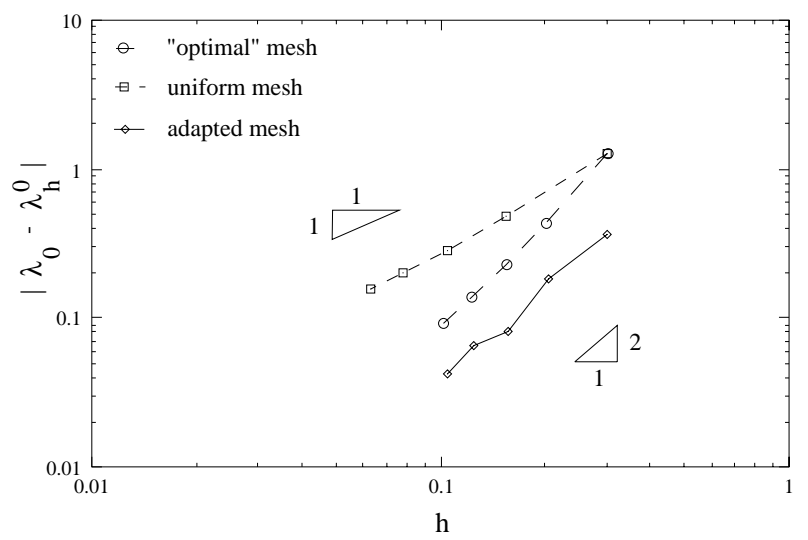

FiguRE 5. Error $\left|\lambda_{0}-\lambda_{h}^{0}\right|$ to $h$, diameter of the mesh, for the computation of the first bifurcation point of (26).

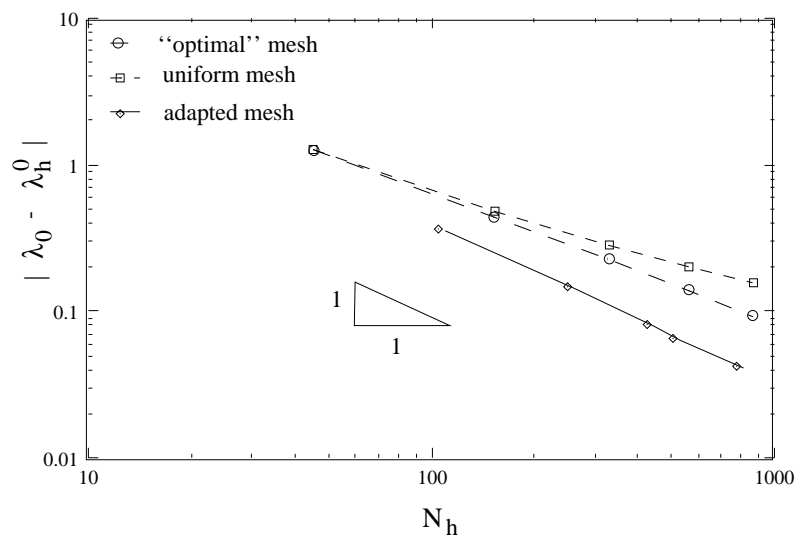

FigURE 6. Error $\left|\lambda_{0}-\lambda_{h}^{0}\right|$ to $N_{h}$, number of vertices of the mesh, for the computation of the first bifurcation point of $(26)$.

\section{REFERENCES}

[1] R.A. Adams, Sobolev spaces. Academic Press (1975).

[2] E.L. Allgower and K. Georg, Numerical continuation methods, an introduction. Springer Verlag, Berlin (1990).

[3] I. Babuška and H.S. Hoo, The $p$-version of the finite element method for domains with corners and for infinite domains. Numer. Meth. PDE 6 (1990) 371-392.

[4] I. Babuška and M. Suri, The h-p version of the finite element method with quasiuniform meshes. RAIRO Modél. Math. Anal. Numér. 21 (1987) 199-238.

[5] H. Brezis, Analyse fonctionnelle. Masson, Paris (1983).

[6] F. Brezzi, J. Rappaz and P.A. Raviart, Finite dimensional approximation of nonlinear problems, part 1: branches of nonsingular solutions. Numer. Math. 36 (1980) 1-36.

[7] F. Brezzi, J. Rappaz and P.A. Raviart, Finite dimensional approximation of nonlinear problems, part 2: limit points. Numer. Math. 37 (1981) 1-28.

[8] , F. Brezzi, J. Rappaz and P.A. Raviart Finite dimensional approximation of nonlinear problems, part 3: simple bifurcation points. Numer. Math. 381981 1-30.

[9] G. Caloz and J. Rappaz, Numerical analysis for nonlinear and bifurcation problems. To appear in Handbook of Numerical Analysis, 1994.

[10] L. Cherfils, Méthode de cheminement adaptative pour les problèmes semi-linéaires dépendant d'un paramètre. Thèse de l'Université J. Fourier, Grenoble I (1996). 
[11] L. Cherfils, Approximation des branches de solutions d'un problème de bifurcation semi-linéaire. C. R. Acad. Sci. Paris 324 (1997) 933-938.

[12] P.G. Ciarlet, Basic error estimates for elliptic problems. In Handbook of numerical analysis. Elsevier Science Publishers B. V., North-Holland (1991).

[13] M. Crouzeix and J. Rappaz, On numerical approximation in bifurcation theory. Masson, Paris (1986).

[14] P. Grisvard, Elliptic problems in nonsmooth domains. Pitman, Boston (1985).

[15] J.C. Paumier, Analyse numérique d'un problème aux limites non linéaire. Numer. Math. 37 (1981) 445-452.

[16] J.C. Paumier, Méthodes numériques pour les bifurcations statiques. collection R.M.A, Masson (1997).

[17] R. Rannacher and R. Scott, Some optimal error estimates for piecewise linear finite element approximations. Math. Comp. 38 (1982) 437-445.

[18] Raugel G., Résolution numérique de problèmes elliptiques dans des domaines avec coins. Thèse de l'Université de Rennes (1978).

[19] P.A. Raviart and J.M. Thomas, Introduction à l'analyse numérique des équations aux dérivées partielles. Masson, Paris (1988).

[20] N.M. Wigley, An efficient method for subtracting off singularities at corners for Laplace's equations. J. Comput. Phys. $\mathbf{7 8}$ (1988) 369-377. 